\title{
Acknowledgment to Referees
}

The editors gratefully acknowledge the valuable contribution of the following referees to the evaluation of the papers submitted to Renal Physiology and Biochemistry

F. Beck, München

G. Burckhardt, Göttingen

G. Capasso, Napoli

M. J. Dunn, Cleveland H. Endou, Tokyo G. Gstraunthaler, Innsbruck W. G. Guder, München D. Häberle, München H. Heinle, Tubingen I. Ichikawa, Nashville D. Kling, Tubingen

F. G. Knox, Rochester

W. Kritz, Heidelberg

A. Kurtz, Zurich

G. Lubec, Wien

H. Murer, Zurich W. Nagel, München H. Oberleithner, Würzburg H. Osswald, Tubingen W. Pfaller, Innsbruck J. Pfeilschifter, Basel J. Puschett, New Orleans E. Schlatter, Freiburg J. Schnermann, Ann Arbor J.S. Schwegler, Würzburg B. Sterzel, Erlangen P. Vina, Montreal N.Walser, Baltimore I. Weiner, Brooklyn 